\title{
Antecedentes de los Procesos Iniciales de Acreditación de los Programas de Especialidades Odontológicas de La Universidad de La Frontera-Chile
}

\author{
Background to the Initial Accreditation Processes For Dental \\ Post-Graduate Programmes at Universidad de La Frontera-Chile
}

\author{
Ramón Fuentes Fernández ${ }^{1}$ \& Claudia Riquelme Macalusso²
}

\begin{abstract}
FUENTES, F. R. \& RIQUELME, M. C. Antecedentes de los procesos Iniciales de acreditación de los programas dee specialidades odontológicas de la Universidad de La Frontera - Chile. Int. J. Odontostomat., 15(2):454-459, 2021.

RESUMEN: Este artículo presenta la experiencia y resultados de la Facultad de Odontología de la universidad de la Frontera al implementar un proceso a-priori de autoevaluación a la creación de los criterios para especialidades odontológicas de la Comisión Nacional de Acreditación (CNA-Chile), para luego entrados en vigencias éstos, se enviaron las tres primeras especialidades odontológicas a procesos de acreditación en Chile. Lo que generó que sea la primera Facultad de Odontología que implementara un Sistema de Gestión de Calidad, impulsando por búsqueda de la mejora continua de sus programas de especialidad.
\end{abstract}

PALABRAS CLAVE: autoevaluación, acreditación, sistema de gestión de calidad.

\section{INTRODUCCIÓN}

Los procesos de autoevaluación hoy en día deben ser una práctica permanente de las instituciones de Educación Superior tanto públicas como privadas; y así ha sido desde la creación de la Ley 20.129 de aseguramiento de la calidad en Chile en el año 2004 $y$ todas sus modificaciones posteriores -lo que ha conllevado a la implementación en los planteles de universitarios una cultura de la evaluación de la calidad considerada como base sine qua non de la mejora continua no tan solo de los programas que se dictan en ellas sino que más bien de la institución mismaestos procesos de autoevaluación han permitido realizar diagnósticos situacionales a partir de la identificación de fortalezas, debilidades, amenazas y oportunidades. En ese sentido, las instituciones de educación superior se han orientado a una búsqueda de calidad en base según Alcántara (2007) en los siguientes aspectos:

- En la satisfacción de las necesidades reales de cada uno de los sectores sociales con quienes interactúan.
- El logro de los objetivos formalmente propuestos.

- La optimización de los recursos.

- Y entender como calidad de la educación superior como un concepto pluridimensional.

Debido a la pluridimensión del concepto de "calidad" en la educación superior desde hace unos veinte años que se ha instalado en el discurso como también en las planificaciones de acción la calidad a través de la búsqueda de la eficiencia y eficacia de los procesos, conceptualizaciones ligadas muy cercanas al mundo ingenieril, sin embargo, las universidades tiene multiplicidad de realidad que hace que la misma institución tenga que estar permanentemente armonizando los ámbitos académicos, sociales y políticos, en donde se debaten puntos de vista distintos y se confrontan acepciones y modelos para lograrla. Por tanto, la calidad es una tendencia hacia la auto-exigencia institucional de manera permanente y con ello avanza en sus metas y objetivos definidos y operacionalizados en planificaciones estratégicas

\footnotetext{
${ }^{1}$ Dental School, Research Centre for Dental Sciences (CICO), Universidad de La Frontera, Temuco, Chile.

${ }^{2}$ Dental School, Universidad de La Frontera, Temuco, Chile.
} 
proyectivas. Asimismo, la calidad entonces debe estar permanentemente en reflexiones y análisis prospectivos y proyectivos, debido que no tan solo se centra en la calidad del sistema educativo, sino que de todas las unidades y componentes que conforman e interactúan en la institución. En ese sentido, para Buendía (2007) el concepto de calidad se plantea en dos dimensiones: 1) intrínseca, la cual se orienta a la búsqueda de la verdad y conocimiento de la realidad educativa institucional y 2) la extrínseca, la cual está focalizada en quienes participan en los procesos e incorpora a la sociedad en general en su desarrollo institucional. Independiente de cada una de ellas y su desarrollo en la institución, ambas buscan alcanzar mayores niveles de calidad, ya que finalmente, el concepto de calidad no se define por sí mismo, sino requiere de ser explicitado en sus componentes o dimensiones no tan solo de las partes sino que un sistema integral e integrado que funciona y opera en relación a fines no tan solo educativos sino que del sistema en general que significa la universidad. Así también, el concepto de calidad estará vinculado de manera permanente a los cambios políticos, económicos y sociales tanto a nivel local como nacional, y es por ello la exigencia externa permanente tanto de los organismos gubernamentales como de las organizaciones civiles del compromiso que puedan desarrollar y vincular con la sociedad. Y es en este último aspecto que la formación de profesionales del área de la salud, y especialmente de la Odontología se da una estrecha interrelación con la calidad, donde a diferencia de la formación tradicional universitaria en el área odontológica los procesos educacionales se fundamentan en la interacción de dos principios: la permanente interconexión entre la teoría y la práctica y del estudio-trabajo clínico, no tan solo en el pregrado sino que en el posgrado (Suárez et al., 2007).

Es por ello, que en los últimos veinte años se ha observado cambios profundos en cómo se entiende la educación odontológica no tan solo en Chile sino que a nivel global; que lo ha conllevado a observar las transformaciones que han experimentado los contenidos y procedimientos de atención en el área, traducido en nuevas dinámicas no tan solo en pregrado sino que también en el posgrado. En Chile es el Ministerio de Salud quien define que se entiende por Especialidad Odontológica, y son las Universidades en sus distintas facultades quienes definen y operacionalizan los elementos necesarios y fundamentales la formación de ellos, por lo cual plantean finalmente como y a quienes quieren formar y especialmente la calidad formativa que quieren entregar.
Para ello la identificación de las variables relevantes para los procesos de evaluación y acreditación de especialidades odontológicas en este caso, responde a una cadena de elementos que interactúan y se entrelazan para la conformación de un sistema de calidad de la formación, como por ejemplo lo realizado por la Facultad de Odontología de la Universidad de La Frontera. La búsqueda de la calidad, está circunscrito masivamente en el pregrado, y el posgrado está supeditado a como se va desarrollando el pregrado en los distintos planteles universitarios; la actividad de postgrado, en general, y dentro de ella la especialización odontológica, se caracteriza antes de los procesos de autoevaluación por una alta pasividad y actualización de sus aspectos curriculares y de gestión, donde los esfuerzos formativos estaban centrados en la adecuada y pertinente relación e interacción profesor-estudiante en el área clínica potente y bien desarrollada en cuanto a conocimientos y prácticas profesionales especializadas. Es por ello que luego de implementados procesos de simulación de autoevaluación de los Programas de Especialidades se logró identificar las variables centrales de los procesos de acreditación, que fueron las bases de la implementación del sistema de aseguramiento de la calidad en la Facultad que se describe a continuación (Otero et al., 2008).

\section{¿Por qué nace el sentido de la autoevaluación para la mejora continua de las especialidades odontológicas de la Universidad de La Frontera?}

El sistema de autoevaluación de las especialidades odontológicas nace el 09 de diciembre de 2013, cuando la Comisión Nacional de Acreditación (CNAChile) a través de la resolución exenta DJ 014-4 se aprueba los criterios de evaluación para la acreditación de especialidades odontológicas de acuerdo a lo dispuesto en la Ley 20.129, y serán tres especialidades de la Universidad de La Frontera quienes ingresen al proceso y sean las primeras en el país en lograr una acreditación (Tabla I). Para la conformación de los criterios de evaluación para la acreditación de especialidades odontológicas, la Comisión Nacional de Acreditación convocó en el mes de agosto del año 2012 la conformación de un Comité Consultivo de Especialidades Odontológicas, a la cual postularon al llamado abierto que realizó la CNA-Chile dos académicos de trayectoria la Facultad de Odontología, quienes aceptados, participaron activamente en las diversas instancias de discusión que se consolido en un documento aprobado en la sesión 686 del día 04 de septiembre de 2013. 
Es así que la Facultad de Odontología de la Universidad pasó a liderar los procesos de autoevaluación de especialidades odontológicas, ya que desde una primera instancia recogió la mirada del ámbito ingenieril para el desarrollo de su posgrado en cuanto a la búsqueda de la calidad educativa que se estaba impartiendo, conformando así un sistema de formación de especialistas odontológico en el sur de Chile de calidad. Para ello realiza dos acciones:

- La incorporación de una profesional del área de las ciencias sociales altamente calificada y con una vasta experiencia en procesos de autoevaluación nacional e internacional en pregrado y posgrado a cargo de la Coordinación de Acreditación, Planificación y Estudios de la Dirección de Calidad Académica de la Facultad y la implementación de un proceso de autoevaluación voluntario a priori a la validación de los criterios para lograr dos objetivos por parte de la Dirección de Calidad Académica y el Decanato de la Facultad para:

1. Mejorar los aspectos administrativos y curriculares previos al proceso formal y legal de acreditación a través de la generación de planes de mejoramiento a un año para ajustar aquellas brechas que fueron necesarias de mejorar.

2. Aproximar a los programas que existían en el años 2013 de especialidades de: Ortodoncia, Odontopediatría y Trastornos Temporo Mandibulares y Dolor Orofacial a los procesos de acreditación.

Proceso de pre-evaluación las especialidades odontológicas de la Universidad de La Frontera en una etapa previa a la creación de los criterios de la CNA-Chile

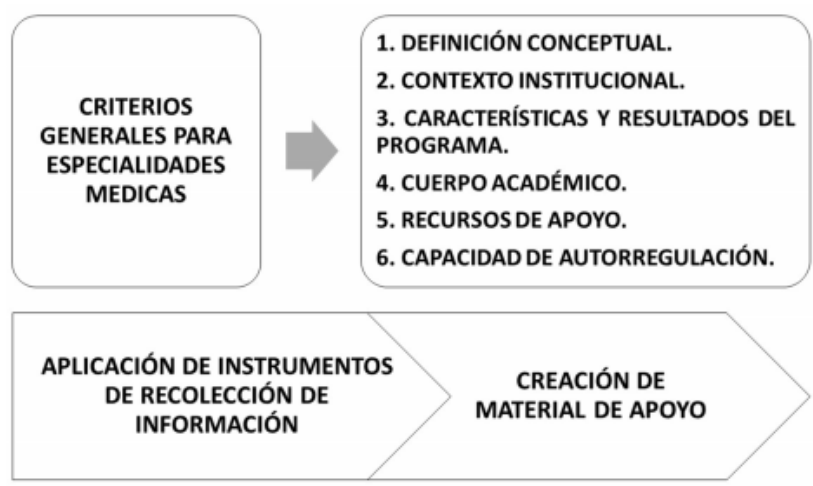

Fig. 1. Homologación de criterios de autoevaluación de especialidades médicas y adaptación de los instrumentos de autoevaluación de especialidades médicas para la recolección de información de las especialidades en odontología de la Universidad de La Frontera- Chile.
En una primera fase se conforma entre la Dirección de Calidad y el Decanato la metodología que se iba a aplicar para llevar a cabo este proceso de pre-evaluación sin criterios para especialidades odontológicas, para ello se recurrió al área más próxima que eran los Criterios Generales para Especialidades Médicas y se realizó una homologación y se adaptaron los instrumentos para lograr analizar la mayor cantidad de aspectos de gestión y curricular de las tres especialidades que participaron en el proceso (Fig. 1).

En una segunda fase se definieron las actividades y los productos asociados a los mecanismos de auto-evaluación, especialmente quienes serían los responsables y las unidades que participaron en la entrega y generación de información cuantitativa y cualitativa para el logro del proceso (Fig. 2). En cuanto a las actividades:

1. Se conformaron los Comités de Autoevaluación con los integrantes de los Comités Académicos de cada uno de los programas, siendo el Director el responsable de los procesos de autoevaluación.

2. Se crea una ruta metodológica (Fig. 3) de acuerdo al contexto y realidad de los equipos que conforman las especialidades de la Facultad de Odontología.

3. Se generan los lazos estratégicos con unidades internas de la Universidad para la generación de información para los análisis internos y externos de los programas.

4. Se definieron los responsables de la conformación de las evidencias y antecedentes que son necesarios para los procesos de autoevaluación.

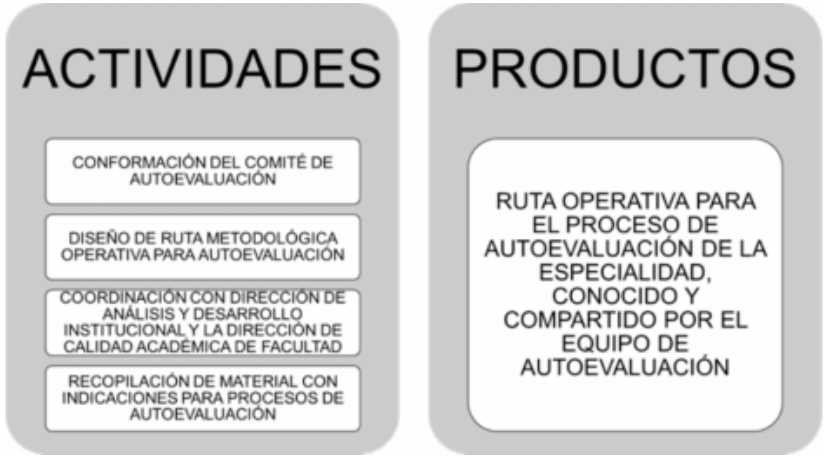

Fig. 2. Homologación de mecanismos de autoevaluación de la Comisión Nacional de Acreditación para el pregrado en las especialidades odontológicas de la Universidad de La Frontera - Chile. 


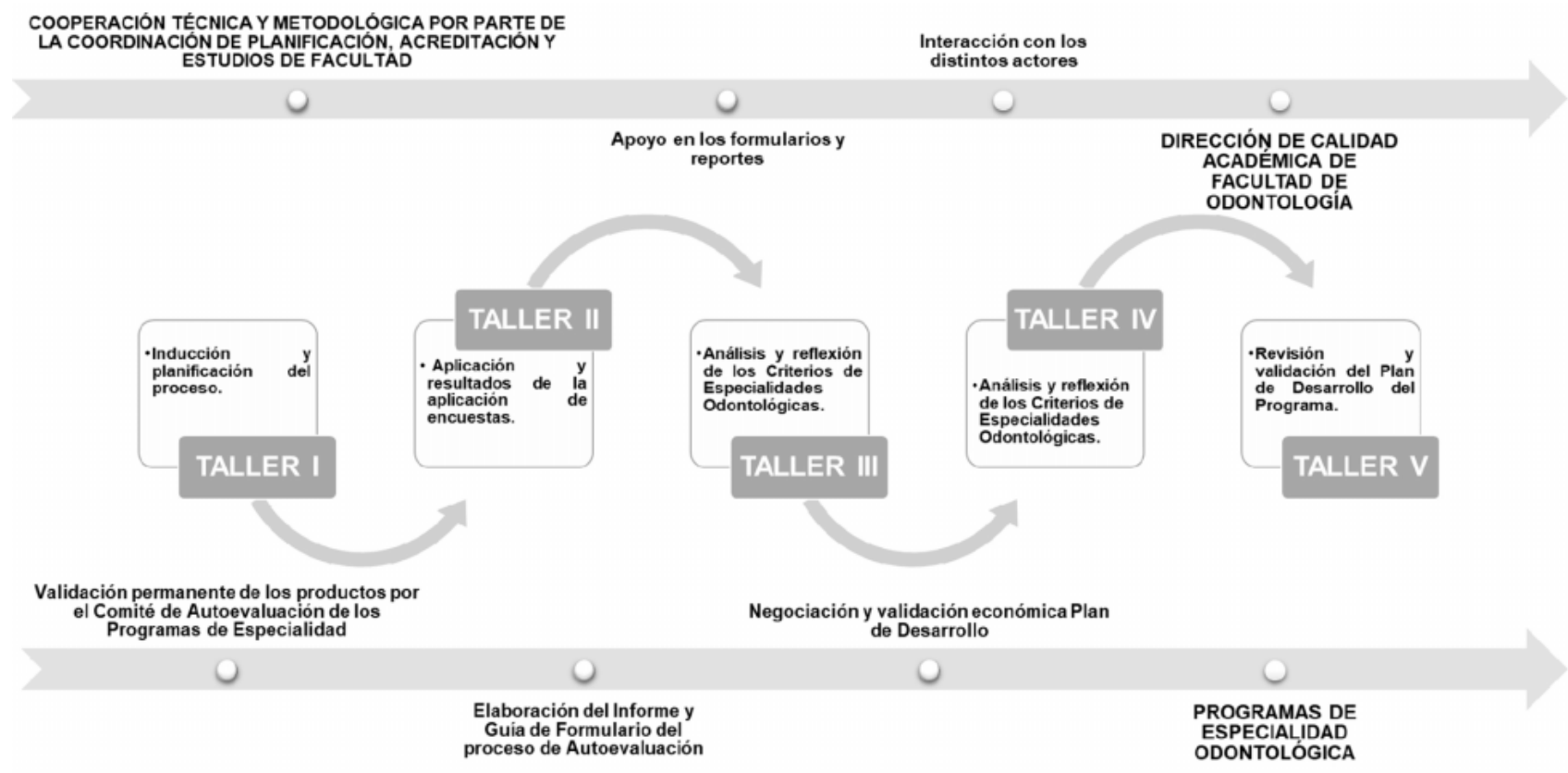

Fig. 3. Etapas del proceso llevado a cabo con las especialidades de los programas existentes en el año 2013-2014 de especialidades odontológicas elaborado por la Coordinación de Planificación, Acreditación y Estudios de la Dirección de Calidad de la Facultad de Odontología de la Universidad de La Frontera - Chile.

Paralelamente, se trabajó con todos los actores que intervienen en estos procesos (Figs. 4 y 5 ), y se les hizo partícipe de todas las instancias de trabajo para que se iniciara el proceso de transformación cultural hacia la mejora continua de sus programas desde quienes dictan las asignaturas como los estudien que confiaron en formarse en la institución.

Como se observa en la Figura 3 se conformó una lógica de jornadas de talleres que durante una jornada de trabajo se lograban abarcar y tratar los aspectos más relevantes del proceso para la obtención y definición de las fortalezas y debilidades del programa, para esos momentos claves del proceso se debe preparar el material previo y la información con la cual se trabajará con los distintos equipos, cada uno de los tres programas tienen lógicas de funcionamientos disimiles debido a aspectos formativos de las especialidades así como también elementos cualitativos que se interrelacionan que van generando particularidades con las cuales en casa uno de los procesos se debe lidiar y adaptar para el logro de los objetivos planteados. Se va trabajando en dos niveles (Comité de Autoevaluación y unidades participantes que apoyan de manera transversal a los procesos) y en tres planos (académicos, estudiantes y titulados).
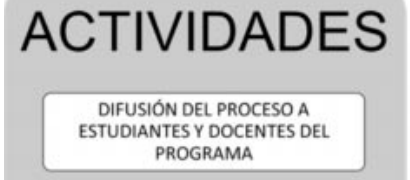

DISEÑO ENCUESTAS IMPRESASY APLICACIÓN DE ENCUESTAS

REUNIONES CON PROFESORES DEL PROGRAMA

\section{PRODUCTOS}

DOCUMENTACIÓN DEL PROGRAMA ORDENADA ACORDE A LAS DIMENSIONES DEL PROCESO DE AUTOEVALUACIÓN
Fig. 4. Recolección de información en las especialidades odontológicas de la Universidad de La Frontera - Chile, existentes en el año 2013-2014.

\section{ACTIVIDADES}

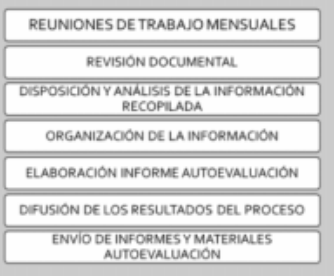

\section{PRODUCTOS}

INFORME AUTOEVALUACION

FORMULARIO DE ESPECIALIDAD

CARPETA DEANEXOS
Fig. 5. Elaboración de los productos homologados para el pre-autoevaluación de los programas existentes en el año 2013-2014 de especialidades odontológicas de la Universidad de La Frontera- Chile. 
FUENTES, F. R. \& RIQUELME, M. C. Antecedentes de los procesos Iniciales de acreditación de los programas dee specialidades odontológicas de la Universidad de La Frontera Chile. Int. J. Odontostomat., 15(2):454-459, 2021.

Tabla I. Resumen de los Programas de Especialidades Odontológicas de la Universidad de La Frontera acreditados.

\begin{tabular}{|c|c|c|c|c|c|}
\hline Programa & Re sultado & Años & $\begin{array}{l}\text { Vigencia } \\
\text { desde }\end{array}$ & $\begin{array}{l}\text { Vigencia } \\
\text { hasta }\end{array}$ & Agencia \\
\hline $\begin{array}{l}\text { Especialidad en Ortodoncia yO rtopedia } \\
\text { Dentomaxilofacial. }\end{array}$ & Acreditado & 3 & $21-10-2015$ & $21-10-2018$ & CNA-Chile \\
\hline Especialidad en Odontopediatría. & Acreditado & 2 & $20-01-2016$ & $20-01-2018$ & CNA-Chile \\
\hline $\begin{array}{l}\text { Especialidad en } \quad \text { Trastornos } \\
\text { Temporomandibulares y Dolor Orofacial. }\end{array}$ & Acreditado & 3 & 08-06-2016 & 08-06-2019 & CNA-Chile \\
\hline
\end{tabular}

Fuente: CNA-Chile.

En definitiva, este proceso de autoevaluación a-priori a la definición de los criterios de autoevaluación de la CNA-Chile para especialidades odontológicas, permitió que cuando se ingresaron las tres especialidades al sistema de acreditación se lograra que acreditaran como las primeras en Chile tanto en sus áreas de especialización como en el registro histórico de la Comisión Nacional de Acreditación (Tabla I).

\section{Aspectos relevantes a considerar para un proce- so de autoevaluación de especialidades odontológicas}

Para el logro en la implementación de un "Sistema para la Gestión de Calidad, este inicia desde el ejercicio de autoevaluación" (Aranda, 2005) en base al análisis, planeación y administración estratégica que permite visualizar, evaluar y formular las estrategias que de acuerdo a lo planteado por para que la calidad no solo sea la eficiencia o eficacia, sino también en la definición cualitativa de esos mismos deseos, expresados en objetivos en pos de la formación de especialistas en odontología como fue el caso de estos tres programas, que para ello se debe:

1. Utilizar una planificación de desarrollo estratégico enfocado a modelos de gestión de mejora continua de las especialidades.

2. La gestión administrativa debe entenderse bajo un esquema de gestión de calidad, observando sus indicadores de desempeño y cumpliendo con las expectativas de los actores internos y externos tanto a nivel de los programas de especialidad como de la facultad a la cual están insertas.

3. Y, adaptarse a los marcos de referencia de las Sociedades Científicas a las cuales se adhieren los académicos y docentes de los programas de especialidad.

Y para que se cumplan todas las etapas del proceso, y en base a lo que plantea Boville et al. (2006) se deben considerar los siguientes aspectos de un proceso de autoevaluación:
1. Considerar que el nivel de disposición para participar en el proceso, es directamente proporcional al grado de información e involucramiento que se tiene sobre el mismo.

2. El ambiente generado por el proceso favorece en el personal el sentido de pertenencia al programa de especialidad y se refleja en su actitud positiva frente a los logros que se obtienen.

3. Es recomendable fomentar una cultura de responsabilidad tanto en los docentes y académicos como en los estudiantes del proceso.

4. Que el Plan de Mejora o Desarrollo sea participativo y responsabilice de las acciones a la mayoría de los participantes.

5. Se debe concienciar la importancia de guardar las evidencias de los avances y progresos de los programas de especialidad.

En síntesis, y de acuerdo a lo planteado por Andión (2007) para que se logre un proceso exitoso de acreditación se debe potenciar y apoyar de manera parmente la(s):

1. Preparación y el compromiso del equipo docente y académico de los programas de especialidad.

2. Disposición y el compromiso de sus estudiantes.

3. Características del modelo educativo de los programas de especialidad odontológicos.

4. Vigencia, pertinencia y relevancia de los Planes de Estudios.

5. Naturaleza de sus servicios de apoyo académico y clínicos.

6. Idoneidad de la infraestructura clínica.

7. Eficacia de su gestión académica.

\section{CONCLUSIONES}

Los procesos de autoevaluación proporcionan una mirada del desempeño de la organización y del grado de madurez del sistema de gestión de la calidad que pueda tener la misma organización; uno de 
los mayores aportes que la identificación de las áreas prioritarias de trabajo para la optimización de los recursos y la mejora continua.

El proceso de autoevaluación en especial en los programas de especialidades odontológicas de la Universidad de La Frontera se ha convertido en gestora principal de la calidad como referente nacional debido a los resultados positivos en sus primeros procesos de acreditación como en el sistema de calidad implementado para el logro de sus objetivos de mejora.

Finalmente, el ejercicio de llevar a cabo un proceso a-priori de autoevaluación de los tres programas de especialidad de la facultad permitió generar una cultura y un clima basado en la búsqueda de la calidad y la mejora de tanto de los procesos de gestión como curricular a través de la reflexión constante de sus fortalezas y debilidades, la implementación de la planificación como mecanismos de seguimiento de los procesos y lo más relevante es que se logró insertar en los programas la idea de la participación conjunta e inclusiva en los procedimientos y procesos de gestión de los mismos programas de especialidad odontológica.

FUENTES, F. R. \& RIQUELME, M. C. Background to the initial accreditation processes for dental post-graduate programmes at Universidad de La Frontera - Chile. Int. J. Odontostomat., 15(2):454-459, 2021.

ABSTRACT: This article presents the experience and results of the Dental School of the La Frontera University, when implementing a-priori process of self-assessment to the creation of the criteria for dental specialties of the CNAChile, the first three dental specialties were sent to accreditation processes in Chile. What generated that it is the first Dental School to implement a Quality Management System, driven by the search for the continuous improvement of its specialty programs.

KEY WORDS: self-evaluation, accreditation, quality management system.

\section{REFERENCIAS BIBLIOGRÁFICAS}

Alcántara, A. Dimensiones de la calidad en educación superior. Reencuentro Univ. Auton. Metrop., 50:21-7, 2007.

Andión, M. Sobre la calidad en la educación superior. Una visión cualitativa. Reencuentro Univ. Auton. Metrop., 50:83-92, 2007. Aranda, H. Resultados obtenidos en la implementación de un sistema para la gestión de calidad en una institución de educación agrícola superior en México. Rev. Mex. Agronegocios, 9(7):111, 2005.
Boville, B.; Argüello, N. \& Reyes, N. La acreditación como proceso dinamizador hacia la calidad. Actual. Investig. Educ., 68(1):121, 2006.

Buendía, A. El concepto de calidad: una construcción en la educación superior. Reencuentro Univ. Auton. Metrop., 50:28-34, 2007.

Otero, J.; Pérez, T. \& Alemañy, E. Una aproximación de las variables a tener en cuenta para la confección de un sistema de acreditación y evaluación de Especialidades. Rev. Habanera Cienc. Med., 7(1):1-12. 2008.

Suárez, L.; Barrios, I. \& González, M. Sistema de evaluación y acreditación de carreras universitarias. Rev. Habanera Cienc. Med., 6(3):1-11, 2007

Corresponding author:

Prof. Dr. Ramón Fuentes Fernández

Research Centre for Dental Sciences

Dental School

Universidad de La Frontera

Av. Francisco Salazar 1145

Temuco

CHILE

E-mail: ramon.fuentes@ufrontera.cl 